\title{
On Legendrian foliations in contact manifolds I: Singularities and neighborhood theorems
}

\author{
YANG HUANG
}

\begin{abstract}
In this note we study several aspects of coisotropic submanifolds of a contact manifold. In particular we give a structure theorem for the singularity of the characteristic foliation of a coisotropic submanifold. Moreover we establish the existence and uniqueness results of germs of contact structures near Legendrian foliations, which is a special case of coisotropic submanifold. This paper can be thought of as an attempt to generalize the study of surfaces in three-dimensional contact geometry to higher dimensions.
\end{abstract}

\section{1. introduction}

A contact manifold $\left(M^{2 n+1}, \xi\right)$ is a closed, oriented manifold with a hyperplane distribution $\xi=\operatorname{ker} \alpha$, where $\alpha$ is a 1-form on $M$ satisfying the condition that $\alpha \wedge(d \alpha)^{n}>0$. Conventionally, we say $M$ is a higher dimensional contact manifold if $n \geq 2$, as opposed to three dimensional contact geometry, which is much better understood currently. In this note we are primarily interested in higher dimensional contact geometry. So although all the results work equally well in dimension three, the conclusions are either trivial or well-known in that case.

For $n=1$ case, it is very important to understand embedded surfaces in $M$, and in particular germs of contact structures on them. There has been an extensive study on these topics in the past few decades. For example the characteristic foliation on surfaces was studied in [2], [3], and convex surface theory was studied in [5].

We wish to generalize our understanding of surfaces in dimension three to higher dimensions as much as possible, and this note can be considered as a small step in that direction. A reasonable analog of surfaces in higher dimension is coisotropic submanifolds, which will be defined in Section 2. Briefly, a submanifold $Y \subset M$ of dimension greater than $n$ is coisotropic if the pointwise intersection $T_{p} Y \cap \xi_{p}$ is a coisotropic subspace of $\xi_{p}$ with 
respect to the conformal symplectic form $d \alpha$, for any $p \in Y$. In this paper we will only consider orientable coisotropic submanifolds unless otherwise specified. Also all the manifolds that are considered in this paper are assumed to be $C^{\infty}$-smooth unless otherwise specified.

A coisotropic submanifold $(Y, \mathcal{F})$ of dimension $n+1$ is called a Legendrian foliation because it naturally comes with a, possibly singular, foliation $\mathcal{F}$ with Legendrian leaves. To state the main results of this paper, we need one more definition. The singular loci $S(Y)$ of a coisotropic submanifold $Y \subset M$ is the set $\left\{p \in Y \mid T_{p} Y \subset \xi_{p}\right\}$. Some previously known constructions, including plastikstufe [9] [12] [4], and bordered Legendrian open books (bLOB) [7], all belong to the category of Legendrian foliations.

The goal of this paper is to establish basic properties of coisotropic submanifolds. The main results are as follows.

Theorem 1.1. Let $Y \subset\left(M^{2 n+1}, \xi\right)$ be a $(n+1)$-dimensional closed orientable coisotropic submanifold and $S(Y)$ be the singular locus of the Legendrian foliation. Then each path-connected component of $S(Y)$, if non-empty, is one of the following

$\left(A_{0}\right):$ A closed orientable $(n-1)$-dimensional submanifold;

$\left(A_{1}\right)$ : An open orientable $(n-1)$-dimensional submanifold;

$\left(A_{2}\right)$ : $A C^{1}$-smooth compact $n$-dimensional (Legendrian) submanifold (which may have nonempty boundary and may not be orientable).

Moreover in the case $\left(A_{0}\right)$ and $\left(A_{1}\right)$, the normal bundle of the $(n-1)$ dimensional submanifold, viewed as a 2-disk bundle, is flat ${ }^{1}$. In the case $\left(A_{2}\right)$, each Legendrian component is isolated from the other singularities, and is equipped with a codimension one foliation with (smooth) isotropic leaves. For Legendrian singularity with boundary, each boundary component is a closed leaf of the coisotropic foliation.

We point out here that by saying $W \subset Y$ is a submanifold, we simply mean each path-connected component of $W$ is smooth manifold itself and the embedding is also smooth. However, we do not assume the embedding is proper, i.e., for a compact subset $K \subset Y, K \cap W$ may not be compact in $W$. For a (open) submanifold $W$ that is not properly embedded, one can nevertheless define its normal bundle, but a neighborhood of the zero section may not be identified with a tubular neighborhood of $W$ in $Y$, unless one

\footnotetext{
${ }^{1}$ The flat structure in general does not respect the linear structure on the normal bundle. See Section 2.2 for more details.
} 
allows the normal radius (with respect to some metric) to shrink to zero at the infinite ends of $W$.

At this moment, however, the structure of $S(Y)$ is more complicated than desired. To prove the neighborhood theorems in this paper, we will rule out case $\left(A_{1}\right)$ and $\left(A_{2}\right)$ by assumption, but for different reasons. In fact, for case $\left(A_{1}\right)$ it is not clear to the author whether the neighborhood theorem exists at all in general. But for case $\left(A_{2}\right)$ one can also prove a neighborhood theorem just like the way we are going to prove Theorem 1.4 below. We will not give the details of the neighborhood theorem in case $\left(A_{2}\right)$, not just because it is similar to (and easier than) case $\left(A_{0}\right)$, but more importantly we believe such (Legendrian) singularities can be either eliminated or turned into $(n-1)$-dimensional singularities, by a small perturbation. Note that although we only have $C^{1}$ smoothness for the Legendrian singularities, it is often enough as far as the (graphical) perturbations are concerned.

Recall that in three-dimensional contact manifolds (i.e., $n=1$ ), our coisotropic submanifold is a surface and the Legendrian foliation is nothing but the characteristic foliation. In this case one can easily perturb the surface so that the characteristic foliation only has isolated singular points. However in dimensions greater than three, the $n$-dimensional singular locus may have nontrivial "inner geometry" according to the last statement in Theorem 1.1, i.e., it comes with a codimension one foliation. This phenomenon is the main source of difficulties that prevent us from easily perturbing them away. Under the assumption that the Legendrian singularity is orientable and the foliation is defined by a closed one-form, it is proved in [10, Proposition I.2.5] that such singularities can be perturbed away by a $C^{\infty}$-small perturbation. More discussion along these lines will be carried out in [6].

Remark 1.2. The constructions of both plastikstufe and bLOB assume that the singular locus has symplectically trivial normal bundle. But in general a flat disk bundle is not necessarily trivial (if $n \geq 4$ ). Even if the bundle is trivial, the flat connection may as well be nontrivial.

Remark 1.3. Since the argument involved in proving Theorem 1.1 is completely local, the conclusions also hold for immersed $Y$.

To state the second result we need some preparations. Let $(Y, \mathcal{F})$ be a $(n+1)$-dimensional manifold with a (singular) codimension one foliation $\mathcal{F}$, and let $S(Y)$ denote the singular locus of $\mathcal{F}$. Briefly speaking, $S(Y)$ is a normally controlled singularity if the following holds:

1) each component of $S(Y)$ is a closed $(n-1)$-submanifold, 
2) the normal bundle of each component of $S(Y)$ is flat,

3 ) for each component of $S(Y)$, there exists a covariant constant Liouville (CCL) 1-form, adapted to $\mathcal{F}$, with respect to the flat connection. See Sections 2.2 and 3.2 for more details.

Note however that a particular choice of a CCL 1-form is not part of the data for normally controlled singularity. We will always assume that $S(Y)$ is co-oriented, i.e., the normal bundle of $S(Y)$ is oriented. Now we state the existence and uniqueness results of germs of contact structures near a Legendrian foliation.

Theorem 1.4. Let $\left(Y^{n+1}, \mathcal{F}\right)$ be a (singularly) foliated manifold such that $\mathcal{F}$ has normally controlled singularity (might be empty). Then the following holds:

- (Existence) There exists a rank $n$ real vector bundle $E$ over $Y$, and a contact structure $\xi$ defined in a neighborhood of the 0-section such that $(Y, \mathcal{F})$ is a Legendrian foliation with respect to $\xi$.

- (Uniqueness) Suppose $Y \subset M^{2 n+1}$ is a submanifold, and $\xi_{0}, \xi_{1}$ are two contact structures on $M$ such that $\mathcal{F}$ is a Legendrian foliation with respect to both contact structures. Then there exists a neighborhood $\mathcal{U}_{i}(Y)$ of $Y, i=0,1$, and a diffeomorphism $\phi: \mathcal{U}_{0}(Y) \rightarrow \mathcal{U}_{1}(Y)$ such that $\phi(Y)=Y$ and $\phi^{*}\left(\xi_{1}\right)=\xi_{0}$.

It might appear that the condition on the singularity of $\mathcal{F}$ is strange. But in fact, it is almost a necessary and sufficient condition for $\mathcal{F}$ to be a Legendrian foliation in some contact manifold, given the assumption that we are in case $\left(A_{1}\right)$ of Theorem 1.1.

Remark 1.5. Singularities in both plastikstufe and bLOB trivially satisfy the conditions in Theorem 1.4. After the first appearance of this paper, it has been brought to the author's attention that similar neighborhood theorems for Legendrian foliations are also known to K. Niederkrüger [10].

Let us briefly discuss here a relatively simple, yet interesting special case of Theorem 1.4. Namely, let $\mathcal{F}$ be a nonsingular codimension one foliation, then the condition on the singularities in Theorem 1.4 is void. It follows that there is a unique (germ of) contact structure with $\mathcal{F}$ as the Legendrian foliation. Following a remarkable result of Thurston [13], a nonsingular codimension one foliation $\mathcal{F}$ exists on $Y$ if and only if the Euler characteristic $\chi(Y)=0$. It is an interesting question to understand the relationship 
between the dynamics of $\mathcal{F}$ and the contact germ. More discussions along these lines will be carried out in a subsequent paper [6]. In particular we will prove the following theorem.

Theorem 1.6. Suppose $(Y, \mathcal{F}) \subset(M, \xi)$ is a coisotropic submanifold such that $\mathcal{F}$ is a nonsingular Legendrian foliation. Then there is a one-to-one correspondence between coisotropic submanifolds $C^{1}$-close to $Y$ in $M$ and nonsingular foliations $C^{1}$-close to $\mathcal{F}$ in $Y$.

It seems that the moduli problem of coisotropic deformations in contact manifolds is, in general, rather complicated, even for nonsingular Legendrian foliations. In fact, the infinitesimal deformations of nonsingular Legendrian foliations already lead us to a twisted version of the tangential de Rham cohomology. More general discussions on deformations of coisotropic submanifolds of arbitrary dimension will also be carried out in [6], and it is interesting to compare our results with the work of Oh-Park [11] in the symplectic setting.

The organization of this note is as follows. In Section 2 we study the characteristic foliation and its singularities of a coisotropic submanifold, and in particular, Legendrian foliations. Then we establish neighborhood theorems for nonsingular and singular Legendrian foliations separately in Section 3. Neighborhood theorem for general coisotrpic submanifolds is also possible but the initial datum are more complicated, so we will postpone the details to [6].

Acknowledgements. The author would like to thank Ko Honda for inviting him to visit Stanford University in 2013, where most material in this paper was firstly presented. Thanks also go to Jian Ge and Thomas Vogel for many inspiring conversations during this work. The author is grateful to the Max Planck Institute for Mathematics in Bonn for providing an excellent environment for research. Last, but not the least, the author thanks an anonymous referee who pointed out several gaps in a previous draft. His suggestions also greatly improved the exposition of the current paper.

\section{The singular loci of Legendrian foliations}

\subsection{Coisotropic submanifolds and singular loci}

Let $\left(M^{2 n+1}, \xi\right)$ be a $(2 n+1)$-dimensional contact manifold, and choose a contact 1-form $\alpha$ such that $\xi=\operatorname{ker} \alpha$. Let $Y^{k} \subset M$ be a closed $k$-dimensional 
submanifold. The case of compact $Y$ with suitable boundary conditions will also be briefly discussed ( $c f$. Lemma 2.14). Define $Y_{\xi}$ to be the (singular) characteristic distribution of $\xi$ in $T Y$, namely, $Y_{\xi}(p)=T_{p} Y \cap \xi_{p}$ for all $p \in Y$. A point $p \in Y$ is singular if $T_{p} Y \subset \xi_{p}$. Let $\lambda=\left.\alpha\right|_{Y} \in \Omega^{1}(Y)$ be the restriction of $\alpha$ to $Y$. Define

$$
S(Y):=\{p \in Y \mid \lambda(p)=0\}
$$

to be the set of singular points in $Y$.

Definition 2.1. A submanifold $Y \subset M$ is coisotropic if for any $p \in Y$, $Y_{\xi}(p) \subset \xi_{p}$ is a coisotropic subspace with respect to the symplectic form $d \alpha$, i.e., $\left(Y_{\xi}(p)\right)^{\perp_{d \alpha}} \subset Y_{\xi}(p)$ where $\perp_{d \alpha}$ is the symplectic orthogonal complement.

It is easy to see that the above definition is independent of the choice of $\alpha$. Moreover, note that by definition $\operatorname{dim}(Y) \geq n$ and $\operatorname{dim}(Y)=n$ if and only if $Y$ is Legendrian and $Y_{\xi}$ is the tangential distribution.

For the rest of this paper, we will be mainly interested in the study of coisotropic submanifold $Y$ of $\operatorname{dim}(Y)=n+1$. In this case Frobenius integrability theorem implies that $Y_{\xi}$ can be integrated to a Legendrian foliation, i.e., a foliation with Legendrian leaves, away from $S(Y)$. In this case, we will call $Y_{\xi}$ the characteristic foliation on $Y$. However the notion of characteristic distribution and characteristic foliation do not coincide in dimensions greater than $n+1$. More details will be discussed in Section 2.3.

The goal of this section is to prove Theorem 1.1. We recall the statement as follows.

Theorem 2.2. Each path-connected component of the singular locus $S(Y) \subset$ $Y$ if nonempty, is one of the following kind.

- A closed orientable $(n-1)$-dimensional submanifold;

- An open orientable $(n-1)$-dimensional submanifold ${ }^{2}$;

- $A C^{1}$-smooth compact n-dimensional (Legendrian) submanifold (which may have nonempty boundary and may not be orientable).

Moreover for the last case, the (compact) Legendrian submanifold $L$ has a collar neighborhood $N(L) \subset Y$ such that $N(L) \cap S(Y)=L$. There also exists a codimension one foliation on $L$ with isotropic leaves. If $\partial L \neq \emptyset$, then each component of $\partial L$ is a closed leaf.

\footnotetext{
${ }^{2}$ Once again note that an open submanifold here is not necessarily properly embedded.
} 
Proof. Suppose $S(Y)$ is nonempty, and therefore there exists $p \in Y$ such that $T_{p} Y \subset \xi_{p}$ is coisotropic. We first work locally in a Darboux chart. It follows from standard Moser's technique that there exists a neighborhood $U(p)$ of $p$ in $M$ contactomorphic to a neighborhood of the origin in $\left(\mathbb{R}^{2 n+1}, \alpha\right)$, where $\alpha=d z-\sum_{i=1}^{n} y_{i} d x_{i}$, such that $p$ is identified with $0 \in \mathbb{R}^{2 n+1}$. Moreover we can assume that $U(p) \cap Y$ is identified with the graph $\Gamma(f)$ of a function

$$
f: \mathbb{R}_{x_{1}, \ldots, x_{n}, y_{n}}^{n+1} \rightarrow \mathbb{R}_{y_{1}, \ldots, y_{n-1}, z}^{n}, \quad \text { where } f(0)=0, d f(0)=0
$$

in a neighborhood of the origin. It is convenient to write

$$
\begin{aligned}
f\left(x_{1}, \ldots, x_{n}, y_{n}\right)= & \left(y_{1}\left(x_{1}, \ldots, x_{n}, y_{n}\right), \ldots, y_{n-1}\left(x_{1}, \ldots, x_{n}, y_{n}\right),\right. \\
& \left.z\left(x_{1}, \ldots, x_{n}, y_{n}\right)\right)
\end{aligned}
$$

Now we construct $n-1$ nonvanishing pointwise linearly independent vector fields in $\mathbb{R}_{x_{1}, \ldots, x_{n}, y_{n}}^{n+1}$ as follows:

$$
\begin{aligned}
\tilde{V}_{1} & =\partial_{x_{1}}-\frac{\partial y_{1}}{\partial y_{n}} \partial_{x_{n}}+\frac{\partial y_{1}}{\partial x_{n}} \partial_{y_{n}}, \\
& \vdots \\
\tilde{V}_{n-1} & =\partial_{x_{n-1}}-\frac{\partial y_{n-1}}{\partial y_{n}} \partial_{x_{n}}+\frac{\partial y_{n-1}}{\partial x_{n}} \partial_{y_{n}},
\end{aligned}
$$

and consider their image in $\Gamma(f)$ given by

$$
\begin{aligned}
V_{k}=f_{*}\left(\tilde{V}_{k}\right)= & \partial_{x_{k}}+\sum_{i=1}^{n-1}\left(\frac{\partial y_{i}}{\partial x_{k}}-\frac{\partial y_{k}}{\partial y_{n}} \frac{\partial y_{i}}{\partial x_{n}}+\frac{\partial y_{k}}{\partial x_{n}} \frac{\partial y_{i}}{\partial y_{n}}\right) \partial_{y_{i}}-\frac{\partial y_{k}}{\partial y_{n}} \partial_{x_{n}} \\
& +\frac{\partial y_{k}}{\partial x_{n}} \partial_{y_{n}}+\left(\frac{\partial z}{\partial x_{k}}-\frac{\partial z}{\partial x_{n}} \frac{\partial y_{k}}{\partial y_{n}}+\frac{\partial z}{\partial y_{n}} \frac{\partial y_{k}}{\partial x_{n}}\right) \partial_{z}
\end{aligned}
$$

for $k=1, \ldots, n-1$. Therefore we have constructed $n-1$ nonvanishing pointwise linearly independent vector fields $\left\{V_{1}, \ldots, V_{n-1}\right\}$ on $\Gamma(f)$ in a neighborhood of the origin, and we claim the following is true.

Claim 2.3. The vector fields $V_{1}, \ldots, V_{n-1}$ satisfies

1) $i_{V_{k}} \alpha=0, \forall k \in\{1, \ldots, n-1\}$

2) $i_{V_{k}} d \lambda=0, \quad \forall k \in\{1, \ldots, n-1\}$, where $\lambda=\left.\alpha\right|_{\Gamma(f)} \in \Omega^{1}(\Gamma(f))$;

3) $\left[V_{k}, V_{l}\right]=0, \forall k, l \in\{1, \ldots, n-1\}$. 
Using Cartan's formula, Claim (1) and (2) imply that

$$
\mathcal{L}_{V_{k}} \lambda=0 .
$$

In other words, the flow of $V_{k}$ preserves $\lambda$ for all $k$. The proof of the claim is a rather tedious but elementary calculation, so we postpone it to the end of the proof and continue to explain how the conclusions of the proposition (partially) follow from the claim. In fact Claim (1) and (3) imply that $\operatorname{span}\left\{V_{1}, \ldots, V_{n-1}\right\}$ can be integrated to a $(n-1)$-dimensional isotropic foliation $\mathcal{H}$ in a neighborhood of the origin in $\Gamma(f)$.

Now (2.1.2) and our assumption that $\lambda$ vanishes at 0 imply that the leaf $S_{0}$ of $\mathcal{H}$ passing through 0 is contained in $S(Y)$. In the following we will only work in an open neighborhood $\mathcal{U}(0)$ of the origin in $\Gamma(f)$. So by abusing notations, we will still denote $S_{0} \cap \mathcal{U}(0)$, which is diffeomorphic to an open ball in $\mathbb{R}^{n-1}$, by $S_{0}$. we identify a tubular neighborhood of $S_{0}$ in $\mathcal{U}(0)$ with its (topologically trivial) normal bundle

$$
\mathbb{R}^{2} \rightarrow E \rightarrow S_{0},
$$

It turns out that $E$ is equipped with a (nonlinear) flat connection such that the horizontal lifts of $S_{0}$ are exactly the leaves of $\mathcal{H}$, and it is such that $\lambda$ is "covariant constant" with respect to the flat connection. In fact such a construction determines the contact structure near the singular loci and will be discussed in more detail in Section 2.2. Since a flat bundle is always locally trivial, by restricting $S_{0}$ to a smaller neighborhood of the origin if necessary, we may assume that $\left.\lambda\right|_{E}$ depends only on the coordinates on the fiber $\mathbb{R}^{2}$. Therefore we may (locally) regard $\lambda$ as a 1 -form on $\mathbb{R}^{2}$. Let $Z(\lambda)=\left\{p \in \mathbb{R}^{2} \mid \lambda(p)=0\right\}$, then $\left.S(Y)\right|_{\mathcal{U}(0)}$ is identified with $Z(\lambda) \times S_{0}$. We have the decomposition $Z(\lambda)=Z_{0} \sqcup Z_{1}$ by dimensions, where $p \in Z_{0}$ if it is itself a path-connected component of $Z(\lambda)$, and $p \in Z_{1}$ if $p$ belongs to an arc which is contained in $Z(\lambda)$.

Now we need to consider the following two cases:

Case 1. Suppose $0 \in Z(\lambda)$ belongs to $Z_{0}$. Then the above discussion implies that $S_{0} \cap \mathcal{U}(0)$ defines a local chart on the leaf $S_{0}$ of $\mathcal{H}$ as a $(n-1)$ dimensional manifold. Moreover $S_{0}$ is either a closed or open submanifold but it cannot have a non-empty boundary. Note, however, that $S_{0} \subset Y$ does not need to be properly embedded as a submanifold because $0 \in Z(\lambda)$ may not be an isolated point, and the points that accumulate at 0 may belong to the same path-connected component as $S_{0}$. The case when $0 \in Z(\lambda)$ is not isolated is rather pathological, and will be excluded from the discussion 
of neighborhood theorems in Section 3.2. Nevertheless let us note that by the flatness of a neighborhood of $S_{0}$ as discussed above, and the assumption that $0 \in Z(\lambda)$ is itself a path-connected component, there can be no other component of $S(Y)$ intersecting transversely or tangentially to $S_{0}$ at 0 .

Case 2. Suppose $0 \in Z(\lambda)$ is contained in $Z_{1}$. Let $I$ be a continuous arc in $Z(\lambda)$ passing through the origin, restricted to a neighborhood of the origin. We first show that $I$ is at least $C^{1}$-smooth at the origin, which will imply immediately that $I$ is a $C^{1}$-smooth arc. Suppose on the contrary that the tangent line to $I$ at 0 does not exist. Then there are at least two rays $R_{1}, R_{2}$ from the origin such that they form a nonzero angle at 0 , and moreover, the intersection $R_{1} \cap I$ (resp. $R_{2} \cap I$ ) contains a sequence of points $\left\{p_{1}, p_{2}, p_{3}, \ldots\right\}$ (resp. $\left\{q_{1}, q_{2}, q_{3}, \ldots\right\}$ ) converging to 0 . This implies $d \lambda=0$ at 0 , which contradicts the contact condition at the origin. So $I$ is indeed a $C^{1}$-smooth arc. Now we see that $I \times S_{0}$ defines a local chart on the pathconnected component of $S(Y)$ containing 0 as a $n$-dimensional manifold, which, unlike the situation in Case 1, can have nonempty boundary. This happens, for example, when $I$ is a half-closed interval.

By the contact condition, it is easy to see that $d \lambda \neq 0$ on each fiber $\mathbb{R}^{2}$ (cf. Lemma 2.9). Let $I \subset Z(\lambda)$ be an arc which passes through 0 . We claimed that there does not exist another arc $I^{\prime} \subset Z(\lambda)$ which intersects $I$ at 0 either transversely or tangentially. The scenario where we have another $I^{\prime}$ intersecting $I$ transversely at 0 is clearly not possible as it will contradict the fact that $d \lambda \neq 0$ at 0 . So suppose otherwise that we have another $I^{\prime}$ tangent to $I$ at 0 . Choosing Cartesian coordinates $x, y$ on $\mathbb{R}^{2}$ such that the $x$-axis is tangent to $I$ (or $I^{\prime}$ ) at the origin. See Figure 1. Using the coordinates we can write

$$
\lambda=A d x+B d y
$$

for functions $A, B \in C^{\infty}\left(\mathbb{R}^{2}\right)$. Let $\left\{x_{1}, x_{2}, x_{3}, \ldots\right\}$ be a decreasing sequence in the $x$-axis such that $\lim _{i \rightarrow \infty} x_{i}=0$, and let $J_{i}$ be the interval between $I$ and $I^{\prime}$ with $x \equiv x_{i}$. These are the dashed lines in Figure 1. Clearly $A$ vanishes on $\partial J_{i}$, and therefore by the Mean Value Theorem, there exists some point $a_{i} \in J_{i}$ such that $\partial_{y} A\left(a_{i}\right)=0$. Since $\lim _{i \rightarrow \infty} a_{i}=0$, by continuity we must have $\partial_{y} A=0$ at the origin. We also have $\partial_{x} B=0$ at the origin by construction. So we arrive at the conclusion that $d \lambda=0$ at the origin, which is a contradiction.

To summarize, we have showed that a path-connected component of $S(Y)$ of dimension $n$ (i.e., Legendrian) cannot intersect another component of $S(Y)$ nor itself. In fact we can easily see that each Legendrian 


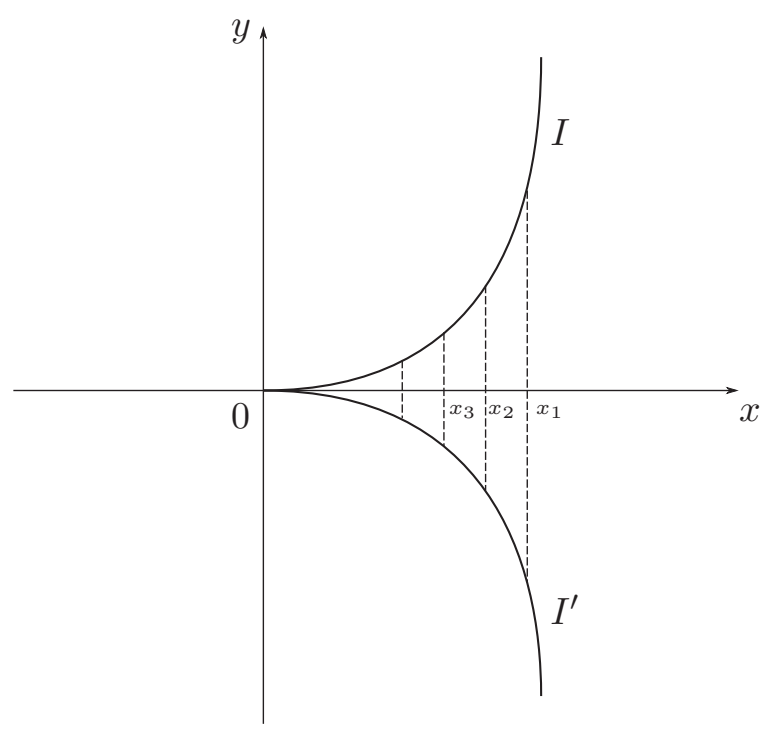

Figure 1: Two arcs $I$ and $I^{\prime}$ meeting tangentially at the origin.

component of $S(Y)$ is either a (properly embedded) closed submanifold or a compact submanifold with boundary as follows. Suppose $L \subset S(Y)$ is a path-connected component, and is itself an open Legendrian submanifold. Consider a path-connected component of $\bar{L} \backslash L$, called $L^{\prime}$. Then $L^{\prime} \subset S(Y)$ since $S(Y)$ is closed. Moreover $L^{\prime}$ is also a Legendrian submanifold because it would be in the same path-connected component of $L$ if its dimension is $n-1$. Then we can find an arc $\gamma$ transversely intersecting $L^{\prime}$ at $p$, such that there is a sequence of points $\left\{p_{1}, p_{2}, p_{3}, \ldots\right\}$ in $\gamma \cap L$ which converges to $p$. See Figure 2. Using an argument similar to the above, we can see that this contradicts the contact condition at $p$.

Note that unlike singular loci of dimension $n-1$ whose orientability is guaranteed by its co-orientability ${ }^{3}$, the $n$-dimensional singular loci is not necessarily orientable. To prove the last statement of Theorem 2.2, we simply observe that the distribution $\operatorname{ker}\left(\left.d \lambda\right|_{L}: T_{L} Y \rightarrow T_{L}^{*} Y\right)$ on $L$ is completely integrable, where $L$ is the (compact) Legendrian singularity. Hence it defines a codimension one foliation $\mathcal{G}$ on $L$, whose leaves are easily see to be isotropic. If $\partial L \neq \emptyset$, then each component of $\partial L$ is a closed leaf of $\mathcal{G}$ essentially by Claim 2.3.

So we have finished the proof of the proposition given Claim 2.3.

\footnotetext{
${ }^{3}$ Recall the nondegenerate 2 -form $d \lambda$ on the normal bundle defines the coorientation.
} 


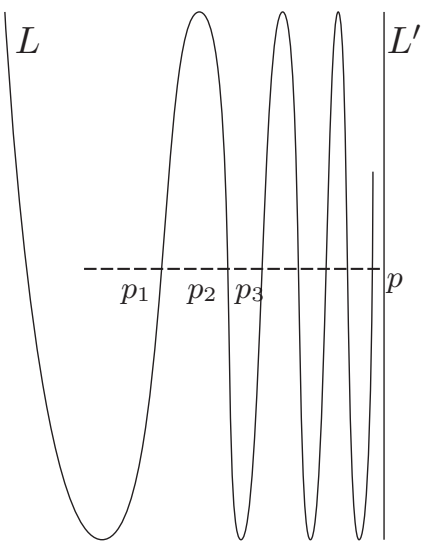

Figure 2: An open Legendrian singularity limiting on another one.

Finally we wrap up the proof of Claim 2.3. An equivalent statement and a simple proof can also be found at [10, Theorem I.1.2]. We keep our lengthy calculations here because it has the virtue of giving explicit spanning vectors, which may be useful at other places.

Proof of Claim (1) and (2). We start by observing that

$$
\begin{aligned}
& T \Gamma(f)=\operatorname{span}\left\{\partial_{x_{1}}+\sum_{i=1}^{n-1} \frac{\partial y_{i}}{\partial x_{1}} \partial_{y_{i}}+\frac{\partial z}{\partial x_{1}} \partial_{z}, \ldots, \partial_{x_{n}}+\sum_{i=1}^{n-1} \frac{\partial y_{i}}{\partial x_{n}} \partial_{y_{i}}+\frac{\partial z}{\partial x_{n}} \partial_{z},\right. \\
& \left.\partial_{y_{n}}+\sum_{i=1}^{n-1} \frac{\partial y_{i}}{\partial y_{n}} \partial_{y_{i}}+\frac{\partial z}{\partial y_{n}} \partial_{z}\right\}
\end{aligned}
$$

By Frobenius integrability theorem and our assumption that $Y_{\xi}$ is a foliation, we have

$$
\begin{aligned}
\left.\alpha \wedge d \alpha\right|_{T \Gamma(f)}= & d z \wedge\left(\sum_{i=1}^{n} d x_{i} \wedge d y_{i}\right) \\
& -\left.\sum_{i=1}^{n}\left(y_{i} d x_{i} \wedge\left(\sum_{j \neq i} d x_{j} \wedge d y_{j}\right)\right)\right|_{T \Gamma(f)}=0
\end{aligned}
$$


which is equivalent to the following set of equations:

$$
\begin{aligned}
& \left(\frac{\partial z}{\partial x_{a}}-y_{a}\right)\left(\frac{\partial y_{b}}{\partial x_{c}}-\frac{\partial y_{c}}{\partial x_{b}}\right)-\left(\frac{\partial z}{\partial x_{b}}-y_{b}\right)\left(\frac{\partial y_{a}}{\partial x_{c}}-\frac{\partial y_{c}}{\partial x_{a}}\right) \\
& +\left(\frac{\partial z}{\partial x_{c}}-y_{c}\right)\left(\frac{\partial y_{a}}{\partial x_{b}}-\frac{\partial y_{b}}{\partial x_{a}}\right)=0, \quad \text { for all } 1 \leq a, b, c \leq n-1 \\
& \left(\frac{\partial z}{\partial x_{a}}-y_{a}\right) \frac{\partial y_{b}}{\partial x_{n}}-\left(\frac{\partial z}{\partial x_{b}}-y_{b}\right) \frac{\partial y_{a}}{\partial x_{n}} \\
& +\left(\frac{\partial z}{\partial x_{n}}-y_{n}\right)\left(\frac{\partial y_{a}}{\partial x_{b}}-\frac{\partial y_{b}}{\partial x_{a}}\right)=0, \quad \text { for all } 1 \leq a, b \leq n-1 \\
& \left(\frac{\partial z}{\partial x_{a}}-y_{a}\right) \frac{\partial y_{b}}{\partial y_{n}}-\left(\frac{\partial z}{\partial x_{b}}-y_{b}\right) \frac{\partial y_{a}}{\partial y_{n}} \\
& +\frac{\partial z}{\partial y_{n}}\left(\frac{\partial y_{a}}{\partial x_{b}}-\frac{\partial y_{b}}{\partial x_{a}}\right)=0, \quad \text { for all } 1 \leq a, b \leq n-1 \\
& \frac{\partial z}{\partial x_{a}}-\left(\frac{\partial z}{\partial x_{n}}-y_{n}\right) \frac{\partial y_{a}}{\partial y_{n}}+\frac{\partial z}{\partial y_{n}} \frac{\partial y_{a}}{\partial x_{n}}-y_{a}=0 \\
& \text { for all } 1 \leq a \leq n-1,
\end{aligned}
$$

which are obtained by evaluating $\alpha \wedge d \alpha$ at all possible choices of three base vectors in $T \Gamma(f)$. Note that (2.1.3) is redundant as it can be deduced from the other three equations but we include it here for completeness.

We first show that $\alpha\left(V_{k}\right)=0, \forall k \in\{1, \ldots, n-1\}$. This follows from a direct calculation as follows.

$$
\begin{aligned}
\alpha\left(V_{k}\right)= & \left(d z-\sum_{i=1}^{n} y_{i} d x_{i}\right)\left(\partial_{x_{k}}+\sum_{i=1}^{n-1}\left(\frac{\partial y_{i}}{\partial x_{k}}-\frac{\partial y_{k}}{\partial y_{n}} \frac{\partial y_{i}}{\partial x_{n}}+\frac{\partial y_{k}}{\partial x_{n}} \frac{\partial y_{i}}{\partial y_{n}}\right) \partial_{y_{i}}\right. \\
& \left.-\frac{\partial y_{k}}{\partial y_{n}} \partial_{x_{n}}+\frac{\partial y_{k}}{\partial x_{n}} \partial_{y_{n}}+\left(\frac{\partial z}{\partial x_{k}}-\frac{\partial z}{\partial x_{n}} \frac{\partial y_{k}}{\partial y_{n}}+\frac{\partial z}{\partial y_{n}} \frac{\partial y_{k}}{\partial x_{n}}\right) \partial_{z}\right) \\
= & \frac{\partial z}{\partial x_{k}}-\frac{\partial z}{\partial x_{n}} \frac{\partial y_{k}}{\partial y_{n}}+\frac{\partial z}{\partial y_{n}} \frac{\partial y_{k}}{\partial x_{n}}-y_{k}+y_{n} \frac{\partial y_{k}}{\partial y_{n}} \\
= & 0
\end{aligned}
$$

where the last equality follows from (2.1.6).

Next we will show that $i_{V_{k}} d \alpha$ vanishes on $T \Gamma(f)$. To this end, we need the following identity

$$
\Lambda_{a, b}:=\frac{\partial y_{a}}{\partial y_{n}} \frac{\partial y_{b}}{\partial x_{n}}-\frac{\partial y_{b}}{\partial y_{n}} \frac{\partial y_{a}}{\partial x_{n}}+\frac{\partial y_{a}}{\partial x_{b}}-\frac{\partial y_{b}}{\partial x_{a}}=0,
$$


for any $1 \leq a, b \leq n-1$. To prove (2.1.7), we plug (2.1.6) into (2.1.4) and (2.1.5) and simplify to get

$$
\left(\frac{\partial z}{\partial x_{n}}-y_{n}\right)\left(\frac{\partial y_{a}}{\partial y_{n}} \frac{\partial y_{b}}{\partial x_{n}}-\frac{\partial y_{b}}{\partial y_{n}} \frac{\partial y_{a}}{\partial x_{n}}+\frac{\partial y_{a}}{\partial x_{b}}-\frac{\partial y_{b}}{\partial x_{a}}\right)=0
$$

and

$$
\frac{\partial z}{\partial y_{n}}\left(\frac{\partial y_{a}}{\partial y_{n}} \frac{\partial y_{b}}{\partial x_{n}}-\frac{\partial y_{b}}{\partial y_{n}} \frac{\partial y_{a}}{\partial x_{n}}+\frac{\partial y_{a}}{\partial x_{b}}-\frac{\partial y_{b}}{\partial x_{a}}\right)=0
$$

respectively. Arguing by contradiction, suppose (2.1.7) is not identically zero. Then by continuity there exists an open set $U$ on which $\Lambda \neq 0$. Therefore we have $\frac{\partial z}{\partial x_{n}}-y_{n}=\frac{\partial z}{\partial y_{n}}=0$ on $U$, but this is impossible because

$$
1=\frac{\partial y_{n}}{\partial y_{n}}=\frac{\partial^{2} z}{\partial y_{n} \partial x_{n}}=\frac{\partial}{\partial x_{n}}\left(\frac{\partial z}{\partial y_{n}}\right)=0
$$

therefore $\Lambda_{a, b}$ must be constantly equal to 0 .

With this preparation, now we can show that

$$
\begin{aligned}
0 & =d \alpha\left(V_{k}, \partial_{x_{l}}+\sum_{i=1}^{n-1} \frac{\partial y_{i}}{\partial x_{l}} \partial_{y_{i}}+\frac{\partial z}{\partial x_{l}} \partial_{z}\right) \\
& =d \alpha\left(V_{k}, \partial_{y_{n}}+\sum_{i=1}^{n-1} \frac{\partial y_{i}}{\partial y_{n}} \partial_{y_{i}}+\frac{\partial z}{\partial y_{n}} \partial_{z}\right)
\end{aligned}
$$

for all $1 \leq l \leq n$. Indeed, the first equality follows from

$$
d \alpha\left(V_{k}, \partial_{x_{l}}+\sum_{i=1}^{n-1} \frac{\partial y_{i}}{\partial x_{l}} \partial_{y_{i}}+\frac{\partial z}{\partial x_{l}} \partial_{z}\right)= \begin{cases}\Lambda_{k, l}=0 & \text { if } 1 \leq l \leq n-1 \\ \frac{\partial y_{k}}{\partial x_{n}}-\frac{\partial y_{k}}{\partial x_{n}}=0 & \text { if } l=n\end{cases}
$$

and the second equality follows from

$$
d \alpha\left(V_{k}, \partial_{y_{n}}+\sum_{i=1}^{n-1} \frac{\partial y_{i}}{\partial y_{n}} \partial_{y_{i}}+\frac{\partial z}{\partial y_{n}} \partial_{z}\right)=\frac{\partial y_{k}}{\partial y_{n}}-\frac{\partial y_{k}}{\partial y_{n}}=0
$$

This completes the proof of Claim (1) and (2).

Proof of Claim (3). Since $\left[V_{k}, V_{l}\right]=\left[f_{*}\left(\tilde{V}_{k}\right), f_{*}\left(\tilde{V}_{l}\right)\right]=f_{*}\left[\tilde{V}_{k}, \tilde{V}_{l}\right]$, it suffices to show $\left[\tilde{V}_{k}, \tilde{V}_{l}\right]=0$ for any $0 \leq k, l \leq n-1$. This is done, again, by explicit 
calculation as follows.

$$
\begin{aligned}
{\left[\tilde{V}_{k}, \tilde{V}_{l}\right] } & =\left[\partial_{x_{k}}-\frac{\partial y_{k}}{\partial y_{n}} \partial_{x_{n}}+\frac{\partial y_{k}}{\partial x_{n}} \partial_{y_{n}}, \partial_{x_{l}}-\frac{\partial y_{l}}{\partial y_{n}} \partial_{x_{n}}+\frac{\partial y_{l}}{\partial x_{n}} \partial_{y_{n}}\right] \\
& =\frac{\partial \Lambda_{k, l}}{\partial y_{n}} \partial_{x_{n}}-\frac{\partial \Lambda_{k, l}}{\partial x_{n}} \partial_{y_{n}}=0,
\end{aligned}
$$

where $\Lambda_{k, l}$ is as defined in (2.1.7).

This completes the proof the proposition.

Remark 2.4. The conclusions of Theorem 2.2 also hold for compact $Y$ with Legendrian boundary ( $c f$. Lemma 2.14).

Note that in the classical theory of surfaces in contact three-manifolds, the characteristic foliation generically only has isolated singular points. This is due to the trivial fact that any small perturbation of an embedded surface is again an embedded surface, and a generic vector field on a surface only possesses isolated singularities. However in dimensions greater than three, a generic perturbation of a coisotropic submanifold will not be coisotropic anymore. In fact, recall from above that if $L \subset S(Y)$ is a Legendrian submanifold, then there is a codimension one foliation $\mathcal{G}$ on $L$ defined by integrating $\operatorname{ker}\left(\left.d \lambda\right|_{L}: T_{L} Y \rightarrow T_{L}^{*} Y\right)$, where $\lambda=\left.\alpha\right|_{Y}$ is the restricted contact form. Here $\mathcal{G}$ may or may not be co-orientable depending on whether $L$ is orientable or not. It is the non-triviality of $\mathcal{G}$ which prevents us from easily perturbing them away. Of course, in dimension three, $\mathcal{G}$ is just the trivial foliation of intervals by points.

\subsection{Germs of contact structure near the singular loci}

In this section we will take a closer look at the (germ of) contact structures in a tubular neighborhood of $S(Y)$ in $Y$. Often we will also write $S$ for $S(Y)$ when the ambient manifold is implicit. For simplicity we will assume in this section that $S$ is a connected closed $(n-1)$-dimensional submanifold of $Y$.

We start by reviewing some standard knowledge on connections on fiber bundles with the setup adapted to our purposes. Readers who are interested in more general theory of connections on fiber bundles are referred to $[1,8]$.

Let

$$
D \rightarrow E \rightarrow B
$$

be a disk bundle over $B$, where $B$ is a closed manifold and $D \subset \mathbb{R}^{2}$ is the unit open disk. We will always abuse notations by identifying $B$ with $B \times\{0\} \subset$ 
$E$ which is the 0 -section of $E$. We say $E$ is vertically oriented if each fiber is oriented.

Remark 2.5. The notion of being vertically oriented is equivalent to the 0-section $B \subset E$ being co-oriented. We will use the term "co-oriented" when we talk about submanifolds.

Remark 2.6. The only reason we do not consider such $E$ as a rank 2 vector bundle is that the parallel transport, to be defined below, is not a linear map in general.

An Ehresmann connection, or connection in brief, $A$ on $E$ is a pointwise decomposition

$$
T_{p} E=V_{p} \oplus H_{p}
$$

which is smoothly varying with $p \in E$, and such that $V_{p}=T_{p} D$ for all $p \in E$, and $H_{p}=T_{p} B$ for all $p \in B$. In the literature, the subbundle $V$ is called the vertical distribution and the subbundle $H$ is called the horizontal distribution. A connection $A$ is called flat if $H$ is completely integrable, ie., the horizontal distribution can be integrated into a codimension two foliation. A bundle $E$ is flat if it admits a flat connection. In this case, let $\mathcal{H}$ be the foliation on $E$ obtained by integrating $H$. In particular $B$ is a closed leaf of $\mathcal{H}$. It is easy to see that all the leaves of $\mathcal{H}$ are transverse to the fibers, and the projection from a leaf to $B$ is a covering map, at least near the 0 -section. We use this observation to define a notion of parallel transport as follows.

Definition 2.7 (Parallel transport). For any path $\gamma:[0,1] \rightarrow B$, the parallel transport along $\gamma$ is a (partially defined) smooth map

$$
\Phi_{\gamma}: E_{\gamma(0)} \rightarrow E_{\gamma(1)}
$$

such that $\Phi_{\gamma}(x)=\tilde{\gamma}(1) \in E_{\gamma(1)}$, where $x \in E_{\gamma(0)}$ and $\tilde{\gamma}:[0,1] \rightarrow E$ is the horizontal lift of $\gamma$ starting at $x$ using the covering map.

Note that since our fiber is not closed, the above parallel transport may not be defined everywhere on $E$, but it is a diffeomorphism whenever it is defined. Since we only care about the parallel transport near the 0 -section, 
we may define the holonomy representation

$$
\mathrm{Hol}: \pi_{1}(B) \rightarrow \operatorname{Diff}_{0}^{+}(D)
$$

using the map $\Phi_{\gamma}$ in Definition 2.7, for any $[\gamma] \in \pi_{1}(B)$. Here we loosely use the symbol $\operatorname{Diff}_{0}^{+}(D)$ to mean the set of orientation-preserving diffeomorphisms from a neighborhood of $0 \in D$ to another (possibly different) neighborhood of $0 \in D$, fixing the origin.

Along the same lines, we define the covariant derivative associated with a flat connection $A$ as follows.

Definition 2.8 (Covariant derivative). Given any vector field $X$ on $B$, we define $\nabla_{X}: \Omega^{k}(E) \rightarrow \Omega^{k}(E)$ by

$$
\nabla_{X} \beta:=\mathcal{L}_{\tilde{X}} \beta
$$

for any $k$-form $\beta \in \Omega^{k}(E)$, where $\tilde{X}$ is the horizontal lift of $X$ on $E$.

With the above preparations, now we consider the singular locus $S \subset Y$, which we assume to be a connected closed submanifold of codimension 2. A tubular neighborhood $N(S) \subset Y$ of $S$ can be identified with a disk bundle over $S$, which we will also denote by $N(S)$. The proof of Theorem 2.2 yields the following observation about the restricted contact form on $N(S)$.

Lemma 2.9. Let $\lambda=\left.\alpha\right|_{N(S)}$ be the restricted contact form as before. Then $N(S)$ is a vertically oriented flat disk bundle on $S$ such that $\lambda$ is covariant constant, i.e., $\nabla_{X} \lambda=0$ for any vector field $X$ on $S$.

Proof. It follows from Claim $2.3(2)$ and (3) that $\operatorname{ker}(d \lambda)$ defines a (codimension 2) foliation $\mathcal{F}$ on $N(S)$ such that $S$ is a closed leaf of $\mathcal{F}$. In particular $d \lambda$ is nondegenerate in the fiber direction and defines an orientation on it. Therefore by definition $N(S)$ is a vertically oriented flat disk bundle with the horizontal distribution given by $\mathcal{F}$. The fact that $\lambda$ is covariant constant with respect to this flat connection follows from (2.1.2).

For later purposes, we also want to look at the converse to Lemma 2.9, i.e., to construct (germs of) contact structures from a flat disk bundle. To this end, we introduce a so-called covariant constant Liouville (CCL) 1-form on $D \subset \mathbb{R}^{2}$ as follows.

Definition 2.10. A 1-form $\beta$ on $D$ is $C C L$ with respect to a flat connection on the disk bundle $D \rightarrow E \rightarrow B$ if the following holds: 
1) $\beta$ is invariant under the action of $\operatorname{Hol}\left(\pi_{1}(B)\right) \subset \operatorname{Diff}_{0}^{+}(D)$,

2) $\beta=0$ exactly at $0 \in D$,

3) $d \beta>0$ on $D$ with respect to the given orientation.

Example 2.11. Suppose $E$ is a trivial flat bundle, namely, the holonomy $\operatorname{Hol}\left(\pi_{1}(B)\right)=\mathrm{id}$. Then $\beta=x d y-y d x$ on $\mathbb{R}^{2}$ is a CCL 1 -form. Following Lemma 2.13 below, one can construct a contact structure on a bundle over $E$ such that $E$ is a Legendrian foliation with a "parameterized elliptic singularity", which shows up in the construction of both plastikstufe and bLOB.

Remark 2.12. In general a CCL 1-form may not exist in a given flat disk bundle. In fact, even a nontrivial covariant constant 1-form does not always exist in general. It is an interesting question to find certain conditions on the holonomy group that guarantees the existence of CCL 1-forms.

Now we state the converse to Lemma 2.9. Let $\pi: \mathcal{E} \rightarrow E$ be a vector bundle on $E$ with fiber $\mathbb{R} \times T^{*} \mathcal{H}$, where $\pi$ is the projection map and $\mathcal{H}$ is the horizontal foliation on $E$. We will denote by $p_{*}: T E \rightarrow T \mathcal{H}$ the horizontal projection map, thinking of $T \mathcal{H}$ as the horizontal distribution of a flat connection.

Lemma 2.13. Suppose $\beta$ is a CCL 1-form as defined above. Then there exists a contact structure $\xi$ on $\mathcal{E}$ such that $E$ is foliated by Legendrians with respect to $\xi$. Moreover $B$ is a co-oriented singular locus in $E$.

Proof. We first construct a 1-form $\eta$ on $\mathcal{E}$ which is similar to the canonical 1form on cotangent bundle as follows. At each $(e, z, v) \in \mathcal{E}$, where $e \in E, z \in$ $\mathbb{R}, v \in T_{e}^{*} \mathcal{H}$, we define $\eta(w):=v\left(p_{*}\left(\pi_{*} w\right)\right)$.

Next we construct a 1 -form $\tilde{\beta}$ on $\mathcal{E}$ by parallel transporting $\beta$ to get a 1 -form $\hat{\beta}$ on $E$, and then pull it back to $\mathcal{E}$ using the projection map $\pi$. Note that $\hat{\beta}$ is globally defined on $E$ by Definition 2.10 (1), and moreover it is covariant constant by construction.

Finally we claim $\alpha:=d z+\tilde{\beta}-\eta$ is a contact form on $\mathcal{E}$ which satisfies all the desired conditions. To check the contact condition, we recall the well-known fact that any flat bundle is locally trivial. So we can choose a (foliated) chart

$$
\phi: \mathbb{R}_{x_{1}, \ldots, x_{n-1}, s, t}^{n+1} \rightarrow U(p) \subset E
$$

for $p \in B$, such that $\phi^{*}(\mathcal{H})$ is the trivial foliation on $\mathbb{R}^{n+1}$ with leaves $\{s=$ const, $t=$ const $\}$. Moreover $\sigma(s, t):=\phi^{*}(\hat{\beta})$ is independent of the $x_{1}, \ldots, x_{n-1}$ 
variables, and $d \sigma$ is nondegenerate in the $(s, t)$-plane. Now $\phi$ induces a chart

$$
\psi: \mathbb{R}_{x_{1}, \ldots, x_{n-1}, s, t, y_{1}, \ldots, y_{n-1}, z}^{2 n+1} \rightarrow \mathcal{U}(p) \in \mathcal{E}
$$

where $y_{1}, \ldots, y_{n-1}$ are the dual coordinates to $x_{1}, \ldots, x_{n-1}$ on $T^{*} \mathcal{H}$, and $z$ is the coordinate on $\mathbb{R}$ as before. It is easy to see that

$$
\psi^{*}(\alpha)=d z+\sigma-\sum_{i=1}^{n-1} y_{i} d x_{i}
$$

is contact in this local chart. Therefore $\alpha$ is a contact form on $\mathcal{E}$.

The assertion that $E$ is foliated by Legendrians and $B$ is a singular locus is now obvious by construction because $\left.\alpha\right|_{E}=\hat{\beta}$.

As an application of our understanding of the restricted contact form near the singular loci, we briefly discuss here the case of compact Legendrian foliated submanifold $Y \subset M$ with Legendrian boundary.

Lemma 2.14. Let $Y \subset M$ be a compact coisotropic submanifold of $M$ with Legendrian boundary. Suppose $S(Y) \subset Y$ is a $(n-1)$-dimensional submanifold. Then each connected component of $S(Y)$ is either contained in $\partial Y$ or contained in the interior of $Y$.

Proof. A connected component of $S(Y)$ cannot be tangent to, but not contained in, $\partial Y$ due to the existence of a horizontal isotropic foliation in a tubular neighborhood. A connected component of $S(Y)$ also cannot transversely intersect $\partial Y$ because if $p \in S(Y) \cap \partial Y$ is a transversal intersection point, then we know from the proof of Lemma 2.9 that $d \lambda$ is nondegenerate on $T_{p} S(Y)^{\perp}$, where $\lambda$ is the restricted contact form. On the other hand, $d \lambda(p)=0$ because $T_{p} S(Y)^{\perp} \subset T_{p}(\partial Y)$ is Legendrian. Therefore we have a contradiction.

\subsection{Coisotropic submanifolds of arbitrary dimension}

Now we consider $k$-dimensional coisotropic submanifold $Y^{k} \subset M^{2 n+1}$ with any $n+1 \leq k \leq 2 n$. In this case, we define the characteristic distribution $\mathcal{F}_{\xi}$ on $Y$ by

$$
\begin{aligned}
\mathcal{F}_{\xi} & =\operatorname{ker}\left(\left.d \alpha\right|_{\xi \cap T Y}\right) \\
& :=\{v \in \xi \cap T Y \mid d \alpha(v, X)=0 \text { for any vector } X \in \xi \cap T Y\} .
\end{aligned}
$$


We will show in Lemma 2.15 that $\mathcal{F}_{\xi}$ is completely integrable, and by an abuse of notations, we will also denote $\mathcal{F}_{\xi}$ the (singular) foliation obtained by integrating the distribution. The singular set of $\mathcal{F}_{\xi}$ is given by $S(Y)=\{p \in$ $\left.Y \mid T_{p} Y \subset \xi_{p}\right\}$. When $k=n+1$, our definition coincides with the definition of Legendrian foliation given at the beginning of Section 2.1 because in that case $\xi \cap T Y \subset \xi$ is Lagrangian. When $k=2 n$, the coisotropy condition is void and $\mathcal{F}_{\xi}$ is a vector field. The well-definedness of $\mathcal{F}_{\xi}$ is the content of the following lemma.

Lemma 2.15. The distribution $\mathcal{F}_{\xi}$ can be integrated to a $(2 n-k+1)$ dimensional foliation away from $S(Y)$.

Proof. Away from $S(Y)$, it is easy to see that $\alpha \wedge d \alpha$ is a nonvanishing form of constant rank, so $\mathcal{F}_{\xi}=\operatorname{ker}\left(\left.d \alpha\right|_{\xi \cap T Y}\right)$ is a $(2 n-k+1)$-dimensional distribution on $Y \backslash S(Y)$. The assertion that $\mathcal{F}_{\xi}$ is integrable is an easy consequence of Frobenius integrability theorem. Namely, let $I^{*}\left(\mathcal{F}_{\xi}\right)=\bigoplus_{i=0}^{\infty} I^{i}\left(\mathcal{F}_{\xi}\right)$ $\subset \Omega^{*}(Y)$ be the annihilator ideal defined by the condition that $\omega \in I^{i}\left(\mathcal{F}_{\xi}\right)$ if and only if $\omega(v) \equiv 0$ for any $v \in \Lambda^{i}\left(\Gamma \mathcal{F}_{\xi}\right)$. It suffices to prove that $d\left(I^{1}\left(\mathcal{F}_{\xi}\right)\right) \subset$ $I^{2}\left(\mathcal{F}_{\xi}\right)$. Note that $I^{1}\left(\mathcal{F}_{\xi}\right)$ is generated by $\left\{\alpha, i_{X} d \alpha, \forall X \in \xi \cap T Y\right\}$. It is clear from definition that $d \alpha \in I^{2}\left(\mathcal{F}_{\xi}\right)$, so it remains to show that $\mathcal{L}_{X}(d \alpha)=$ $d\left(i_{X} d \alpha\right) \in I^{2}\left(\mathcal{F}_{\xi}\right)$. To this end, let $Y_{1}, Y_{2} \in \Gamma \mathcal{F}_{\xi}$ be two vector fields tangent to $\mathcal{F}_{\xi}$. Then

$$
\mathcal{L}_{X}(d \alpha)\left(Y_{1}, Y_{2}\right)=X\left(d \alpha\left(Y_{1}, Y_{2}\right)\right)-d \alpha\left(\mathcal{L}_{X} Y_{1}, Y_{2}\right)-d \alpha\left(Y_{1}, \mathcal{L}_{X} Y_{2}\right)=0,
$$

where the last equation follows from the fact that $\mathcal{L}_{X} Y_{i} \in \xi \cap T Y$ for $i=1,2$, since $\alpha\left(\mathcal{L}_{X} Y_{i}\right)=\alpha\left(\left[X, Y_{i}\right]\right)=X\left(\alpha\left(Y_{i}\right)\right)-Y_{i}(\alpha(X))-d \alpha\left(X, Y_{i}\right)=0$.

We now give a statement on the dimension of $S(Y)$ for any coisotropic $Y$, similar to Theorem 2.2. However since the normal bundle to the singular loci of high codimension is even more complicated than the Legendrian foliation case, we will not attempt to describe the behavior of the characteristic foliation in the normal directions as we did for the Legendrian foliation case. In fact we will omit the calculations involved in the proof the following proposition, because it is lengthy and rather similar to the one we used to prove Claim 2.3. The interested reader may also try to find a coordinate-free proof using the ideas similar to the proof of [10, Theorem I.1.2].

Proposition 2.16. Given a closed coisotropic submanifold $Y$ of dimension $k, n+1 \leq k \leq 2 n$. For each point $p \in S(Y)$, there is a $(2 n-k)$-dimensional (not necessarily properly embedded) submanifold without boundary which passes through $p$ and is contained in $S(Y)$. 
Sketch of proof. The proof is essentially the same as the proof of Theorem 2.2, so we only give a sketch here. Now $Y$ in a neighborhood of a singular point $p \in S(Y)$ is modeled on the graph of a function

$$
f: \mathbb{R}_{x_{1}, \ldots, x_{n}, y_{2 n-k+1}, \ldots, y_{n}}^{k} \rightarrow \mathbb{R}_{y_{1}, \ldots, y_{2 n-k}, z}^{2 n-k+1}, \quad \text { such that } f(0)=0, d f(0)=0,
$$

in the standard $\left(\mathbb{R}^{2 n+1}, d z-\sum_{i=1}^{n} y_{i} d x_{i}\right)$. This is analogous to (2.1.1). All the calculations thereafter carries over to this case with little modification, so we leave the details to the interested reader.

\section{Neighborhood theorems for Legendrian foliations}

\subsection{The case of nonsingular Legendrian foliations}

We will show the existence and uniqueness of the germ of a contact structure near a closed (sub-)manifold with fixed nonsingular (Legendrian) foliation. To formulate the existence part more precisely, let $\left(Y^{n+1}, \mathcal{F}\right)$ be a closed foliated manifold of dimension $n+1$, where $\mathcal{F}$ is a co-oriented codimension one foliation. Consider a vector bundle $E \rightarrow Y$ with fiber $T^{*} \mathcal{F}$, then we have the following

Lemma 3.1 (Existence). There is a contact structure $\xi$ on the total space of $E$ such that $\mathcal{F}$ is the characteristic foliation on $Y$.

Proof. The technique for constructing the contact structure is similar to the one used in the proof of Lemma 2.13. But here we need one more piece of data, namely, a line field $L$ on $Y$ transverse to $\mathcal{F}$. Then we have $T Y=T \mathcal{F} \oplus$ $L$. Let $p_{1}: T Y \rightarrow T \mathcal{F}$ be the projection to the first factor. Let $\pi: E \rightarrow Y$ be the projection map. We define a "tautological 1-form" $\eta$ on $E$ as follows. For any $v \in T_{(p, w)} E$, where $p \in Y, w \in T_{p}^{*} \mathcal{F}$, define $\eta(v):=w\left(p_{1}\left(\pi_{*} v\right)\right)$.

Now let $\beta \neq 0 \in \Omega^{1}(Y)$ be a defining 1 -form of $\mathcal{F}$, i.e., $\operatorname{ker} \beta=\mathcal{F}$. Abusing notations, we will also write $\beta$ for $\pi^{*}(\beta) \in \Omega^{1}(E)$. Then we claim that $\alpha=\beta-\eta$ is a contact form on $E$. To see this, we compute locally by choosing a (foliated) chart

$$
\phi: \mathbb{R}_{t, x_{1}, \ldots, x_{n}}^{n+1} \rightarrow \mathcal{U}(p) \in Y
$$

near some point $p \in Y$, such that the leaves of $\mathcal{F}$ are given by $\{t=$ const $\}$. Let $y_{i}$ be the dual coordinates to $x_{i}$ on $T^{*} \mathcal{F}$. Suppose that locally $L$ is 
spanned by the vector field

$$
L=\left\langle\partial_{t}+\sum_{i=1}^{n} R_{i} \partial_{x_{i}}\right\rangle
$$

where $R_{i} \in C^{\infty}(\mathcal{U}(p))$ for $1 \leq i \leq n$.

Locally we may write $\beta=f d t$ for some $f>0 \in C^{\infty}(\mathcal{U}(p))$. One easily sees that in these coordinates

$$
\eta=-\left(\sum_{i=1}^{n} R_{i} y_{i}\right) d t+\sum_{i=1}^{n} y_{i} d x_{i} .
$$

So we have

$$
\alpha=\left(f+\sum_{i=1}^{n} R_{i} y_{i}\right) d t-\sum_{i=1}^{n} y_{i} d x_{i} .
$$

Verifying $\alpha$ is indeed a contact form is a straightforward calculation which we will carry out explicitly as follows.

$$
\begin{aligned}
& \alpha \wedge(d \alpha)^{n} \\
= & \left(\left(f+\sum_{i=1}^{n} R_{i} y_{i}\right) d t-\sum_{i=1}^{n} y_{i} d x_{i}\right) \\
& \wedge\left(\sum_{j=1}^{n}\left(\frac{\partial f}{\partial x_{j}}+\sum_{i=1}^{n} \frac{\partial R_{i}}{\partial x_{j}} y_{i}\right) d x_{j} \wedge d t+\sum_{i=1}^{n} R_{i} d y_{i} \wedge d t+\sum_{i=1}^{n} d x_{i} \wedge d y_{i}\right)^{n} \\
= & n !\left(\left(f+\sum_{i=1}^{n} R_{i} y_{i}\right) d t-\sum_{i=1}^{n} y_{i} d x_{i}\right) \wedge\left(d x_{1} \wedge d y_{1} \wedge \cdots \wedge d x_{n} \wedge d y_{n}\right. \\
& +\sum_{j=1}^{n}\left(\frac{\partial f}{\partial x_{j}}+\sum_{i=1}^{n} \frac{\partial R_{i}}{\partial x_{j}} y_{i}\right) d x_{1} \wedge d y_{1} \wedge \cdots \wedge d x_{j} \wedge \widehat{d y_{j}} \wedge \cdots \wedge d x_{n} \wedge d y_{n} \wedge d t \\
& \left.+\sum_{i=1}^{n} R_{i} d x_{1} \wedge d y_{1} \wedge \cdots \wedge \widehat{d x_{i}} \wedge d y_{i} \wedge \cdots \wedge d x_{n} \wedge d y_{n} \wedge d t\right) \\
= & n !\left(f+\sum_{i=1}^{n} R_{i} y_{i}-\sum_{i=1}^{n} R_{i} y_{i}\right) d \mathrm{vol} \\
= & n ! f d \mathrm{vol}>0 .
\end{aligned}
$$

Here $\widehat{ }$ means the corresponding term is missing, and $d$ vol $=d x_{1} \wedge d y_{1} \wedge$ $\cdots \wedge d x_{n} \wedge d y_{n} \wedge d t$. 
Finally the assertion that $\mathcal{F}$ is the characteristic foliation with respect to $\xi=\operatorname{ker} \alpha$ follows from the construction.

It turns out that the contact structure $\xi$ constructed in Lemma 3.1 is, in fact, uniquely determined by $\mathcal{F}$ up to contactomorphism. This is the content of the following lemma and its proof mimics the proof of Weinstein neighborhood theorem.

Lemma 3.2 (Uniqueness). Let $(Y, \mathcal{F}) \subset M$ be a foliated submanifold. Suppose $\xi_{0}, \xi_{1}$ are contact structures on $M$ such that $\mathcal{F}$ is a Legendrian foliation with respect to both $\xi_{0}$ and $\xi_{1}$. Then there exists neighborhoods $\mathcal{U}_{0}$ and $\mathcal{U}_{1}$ of $Y$, and a diffeomorphism $\phi: \mathcal{U}_{0} \rightarrow \mathcal{U}_{1}$ such that (1) $\left.\phi\right|_{Y}=\operatorname{Id}_{Y}$; (2) $\phi_{*}\left(\xi_{0}\right)=\xi_{1}$.

We first recall the Whitney extension theorem as follows.

Theorem 3.3 (Whitney Extension Theorem). Let $Y \subset M$ be a submanifold. Suppose there is a pointwise linear isomorphism $L_{p}: T_{p} M \rightarrow T_{p} M$, depending smoothly on $p \in Y$, such that $\left.L_{p}\right|_{T_{p} Y}=\operatorname{Id}_{T_{p} Y}$. Then there exists an embedding $h: \mathcal{U} \rightarrow M$ of some neighborhood $\mathcal{U}$ of $Y$ in $M$ such that $\left.h\right|_{Y}=\operatorname{Id}_{Y}$ and $d h_{p}=L_{p}$ for all $p \in Y$.

Proof of Lemma 3.2. Choose a contact form $\alpha_{i}$ for $\xi_{i}, i=0,1$, and a Riemannian metric $g$ on $M$. Let $X$ be a nonvanishing vector field on $Y$ transverse to $\mathcal{F}$. Rescaling $\alpha_{i}$ if necessary, we can assume $\alpha_{0}(X)=\alpha_{1}(X)$.

Now we define a pointwise linear isomorphism $L_{p}: T_{p} M \rightarrow T_{p} M$ for each $p \in Y$ in the following two steps.

Step 1: Let $\left.L_{p}\right|_{T_{p} Y}=\operatorname{Id}_{T_{p} Y}$.

Step 2: Consider the orthogonal decomposition

$$
\xi_{i, p}=T_{p} \mathcal{F} \oplus\left(T_{p} \mathcal{F}\right)^{\perp_{i}}, i=0,1
$$

with respect to $g$, and notice that $T_{p} \mathcal{F}$ is a Lagrangian subspace in both $\xi_{0}$ and $\xi_{1}$. Choose a basis on $T_{p} \mathcal{F}=\operatorname{span}\left\{e_{1}, \ldots, e_{n}\right\}$, then a simple symplectic linear algebra implies that we can canonically complete the $e_{i}$ 's to a symplectic basis

$$
\begin{aligned}
& \xi_{0, p}=\operatorname{span}\left\{e_{1}, \ldots, e_{n}, f_{1}, \ldots, f_{n}\right\} \\
& \xi_{1, p}=\operatorname{span}\left\{e_{1}, \ldots, e_{n}, g_{1}, \ldots, g_{n}\right\}
\end{aligned}
$$


in the sense that $d \alpha_{0}\left(e_{i}, f_{j}\right)=d \alpha_{1}\left(e_{i}, g_{j}\right)=\delta_{i j}$ and all the other pairings vanish, where $\left(T_{p} \mathcal{F}\right)^{\perp_{0}}=\operatorname{span}\left\{f_{1}, \ldots, f_{n}\right\}$ and $\left(T_{p} \mathcal{F}\right)^{\perp_{1}}$ $=\operatorname{span}\left\{g_{1}, \ldots, g_{n}\right\}$. Now let $L_{p}\left(f_{i}\right)=g_{i}$ for $1 \leq i \leq n$.

It is easy to check that the definition of $L_{p}$ is independent of the choice of a basis on $T_{p} \mathcal{F}$, and $L_{p}$ is smoothly varying with $p$ because the symplectic basis completion is canonical. Therefore Theorem 3.3 produces a diffeomorphism $\psi: \mathcal{U}_{0} \rightarrow \mathcal{U}_{1}$ between neighborhoods of $Y$ which is fixed on $Y$ such that $\psi^{*}\left(\alpha_{1}\right)=\alpha_{0}$ and $\psi^{*}\left(d \alpha_{1}\right)=d \alpha_{0}$ on $Y$.

Finally a standard Moser's technique in a (possibly smaller) neighborhood of $Y$ isotopes $\psi$ to $\phi: \mathcal{U}_{0} \rightarrow \mathcal{U}_{1}$ which satisfies all the desired properties.

Remark 3.4. In fact the conclusions of Lemma 3.2 still hold true if we allow $(Y, \mathcal{F})$ to be embedded in two different contact manifolds $\left(M_{1}, \xi_{1}\right)$ and $\left(M_{2}, \xi_{2}\right)$, as is done in [10]. The point is to observed that the normal bundles on $Y$, viewed as submanifolds in $M_{1}$ and $M_{2}$ respectively, must be isomorphic to each other.

Remark 3.5. Another possible generalization of Lemma 3.2 is to allow $Y$ to be compact with boundary. The is because the Whitney extension theorem only uses the exponential maps in the normal directions to $Y$, and it does not require $Y$ to be closed. The rest of the proof goes through without any changes.

\subsection{The case of singular Legendrian foliations}

Due to our lack of complete knowledge on the singular loci of Legendrian foliations, we will assume for the rest of this section that

$$
S(Y)=S_{1} \cup \cdots \cup S_{k} \subset Y
$$

is a finite disjoint union of $(n-1)$-dimensional closed co-oriented submanifolds. Recall that a codimension 1 singular foliation $\mathcal{F}=\operatorname{ker} \beta$ in $Y$ has normally controlled singularities if the singular locus $S(Y)$ is a finite union of $(n-1)$-dimensional closed co-oriented submanifolds, and a tubular neighborhood of each path-connected component of $S(Y)$ has the structure of a flat disk bundle ( $c f$. Section 2.2) with respect to which the restriction of $\beta$ to the fiber direction is a CCL 1-form in the sense of Definition 2.10.

With the above assumption in mind, we present the existence of a germ of contact structure with given singular foliation as follows. 
Lemma 3.6 (Existence). Suppose $(Y, \mathcal{F})$ is foliated manifold with normally controlled singularities. Then there exists a vector bundle $E \rightarrow Y$ and a contact structure $\xi$ on a neighborhood of the 0-section such that $\mathcal{F}$ is the characteristic foliation on $Y$.

Proof. The proof is just assembling several pieces from previous sections together. Namely, we decompose

$$
Y=N\left(S_{1}\right) \cup \cdots \cup N\left(S_{k}\right) \cup Q
$$

where $N\left(S_{i}\right)$ are tubular neighborhoods of each component of $S(Y)$, and $Q$ is the part of $Y$ where $\mathcal{F}$ is nonsingular. We assume that $Q$ intersects each $N\left(S_{i}\right)$ in a collar neighborhood of $\partial N\left(S_{i}\right)$.

Over each $N\left(S_{i}\right)$, Lemma 2.13 produces a vector bundle $E_{i} \rightarrow N\left(S_{i}\right)$ with fiber $\mathbb{R} \times T^{*} \mathcal{H}_{i}$, where $\mathcal{H}_{i}$ is the horizontal foliation on $N\left(S_{i}\right)$, together with a contact structure $\xi_{i}$ on the total space such that $\left.\mathcal{F}\right|_{N\left(S_{i}\right)}$ is the Legendrian foliation. Over $Q$, Lemma 3.1 produces a vector bundle $E_{0} \rightarrow Q$ with fiber $T^{*} \mathcal{F}$ and a contact structure $\xi_{0}$ on the total space such that $\left.\mathcal{F}\right|_{Q}$ is the Legendrian foliation.

We only need to observe that the above pieces of bundles and contact structures can be patched together, thanks to Lemma 3.2 and the remarks right after it (applied to the overlap between $Q$ and each $N\left(S_{i}\right)$ ). Finally we note that the gluing map is in general not fiberwise linear due to the application of Moser's technique in Lemma 3.2, so to be honest, we will get a fiber bundle with fiber $\mathbb{R}^{n}$ after patching. But this is a vector bundle since $\operatorname{Diff}_{0}\left(\mathbb{R}^{n}\right)$ deformation retracts onto $\operatorname{GL}(n, \mathbb{R})$.

Remark 3.7. Unlike Lemma 3.1, the contact structure constructed in Lemma 3.6 is only defined near the 0 -section.

Now we present the uniqueness of the germ of contact structure constructed in Lemma 3.6, which is analogous to Lemma 3.2. But due to the presence of singularities, we cannot expect the diffeomorphism to be the identity on $Y$ anymore.

Lemma 3.8 (Uniqueness). Let $(Y, \mathcal{F}) \subset M$ be a singularly foliated submanifold with co-oriented singularities. Suppose $\xi_{0}, \xi_{1}$ are contact structures on $M$ such that $\mathcal{F}$ is a Legendrian foliation with respect to both $\xi_{0}$ and $\xi_{1}$. Then there exists neighborhoods $\mathcal{U}_{0}$ and $\mathcal{U}_{1}$ of $Y$, and a diffeomorphism $\phi: \mathcal{U}_{0} \rightarrow \mathcal{U}_{1}$ such that (1) $\phi(Y)=Y$; (2) $\phi_{*}\left(\xi_{0}\right)=\xi_{1}$. 
Proof. Let $\alpha_{0}, \alpha_{1}$ be contact forms for $\xi_{0}, \xi_{1}$ respectively, and $g$ be a Riemannian metric on $M$. For the sake of simplicity, we assume the singular locus $S$ of $\mathcal{F}$ is connected, and write $Y=N_{\epsilon}(S) \cup Q$ be the decomposition as before, such that $Q$ intersects $N_{\epsilon}(S)$ in an arbitrarily small neighborhood of $\partial N_{\epsilon}(S)$. Here $N_{\epsilon}(S)$ is a tubular neighborhood of $S$ of radius $\epsilon>0$, a small constant which will be determined later. Let $\lambda_{i}=\left.\alpha_{i}\right|_{Y}, i=0,1$, be the restricted contact form. We first claim that up to a contact isotopy of $\left(M, \xi_{1}\right)$ which fixes $Y$, we can assume $\lambda_{0}=\lambda_{1}$.

Away from $S$, it is clear that $\lambda_{1}=\mu \lambda_{0}$ for some nonzero function $\mu$, but it is not so easy to extend $\mu$ over $S$. So we use Moser's technique instead as follows. As usual, we identify $N_{\epsilon}(S)$ with the normal (plane) bundle $E$ on $S$ in $Y$. Let $\lambda_{t}=(1-t) \lambda_{0}+t \lambda_{1}, 0 \leq t \leq 1$ be a path of 1 -forms connecting $\lambda_{0}$ and $\lambda_{1}$. It is clear that $\operatorname{ker} \lambda_{t}=\operatorname{ker} \lambda_{0}=\operatorname{ker} \lambda_{1}=\mathcal{F}$ for all $t$. Moreover $d \lambda_{t}$ is nondegenerate in the fiber directions of $E$. This is because $d \lambda_{0}$ and $d \lambda_{1}$ define the same co-orientation of $S$ by assumption. It follows from the proof of Lemma 2.9 that $\mathcal{H}_{t}=\operatorname{ker}\left(d \lambda_{t}\right)$ defines (the horizontal lifts of) a flat connection on $E$. We therefore have a pointwise splitting $T E=\mathbb{R}^{2} \oplus T \mathcal{H}_{t}$ which depends on time $t$. Here $\mathbb{R}^{2}$ stands for the fiber (or vertical) direction. Following the standard Moser's technique, we only need to find a timedependent vector field $Y_{t}$ such that

$$
\mathcal{L}_{Y_{t}} \lambda_{t}=d\left(i\left(Y_{t}\right) \lambda_{t}\right)+i\left(Y_{t}\right) d \lambda_{t}=\frac{d \lambda_{t}}{d t}=\lambda_{1}-\lambda_{0}
$$

Now by the nondegeneracy of $d \lambda_{t}$, there is a unique vector field $Y_{t}$, tangent to the fiber direction, such that $i\left(Y_{t}\right) d \lambda_{t}=\lambda_{1}-\lambda_{0}$. Here we used the fact that $\lambda_{1}-\lambda_{0}$ vanishes in directions tangent to $\mathcal{H}_{t} \subset \mathcal{F}$, so does $d \lambda_{t}$ by construction. Finally we notice that such $Y_{t}$ must be tangent to $\mathcal{F}$, again, by the nondegeneracy of $d \lambda_{t}$. So we may conclude that the above constructed $Y_{t}$ satisfies (3.2.2). There is no problem in extending $Y_{t}$ to a (time dependent) vector field on $M$, whose time-1 flow gives a diffeomorphism $\phi: M \rightarrow M$. It is such that $\phi(Y)=Y$ and $\lambda_{1}^{\prime}:=\left.\phi^{*}\left(\alpha_{1}\right)\right|_{Y}=\lambda_{0}$, as claimed.

Hereafter we will assume $\lambda_{0}=\lambda_{1}$.

Let $X_{i}$ be the unit normal vector field to $\xi_{i}$ for $i=0,1$. We define a pointwise linear isomorphism $L_{p}: T_{p} M \rightarrow T_{p} M$ for each $p \in Y$ in three steps as follows.

Step 1: Let $\left.L_{p}\right|_{T_{p} Y}=\operatorname{Id}_{T_{p} Y}$.

Step 2: We define $L_{p}$ for $p \in Q$. Orthogonally decompose

$$
\xi_{i, p}=T_{p} \mathcal{F} \oplus\left(T_{p} \mathcal{F}\right)^{\perp_{i}}, i=0,1,
$$


with respect to the metric $g$ as before. The same argument used in Lemma 3.2 defines a map $L_{p}\left(f_{i}\right)=g_{i}$ for $1 \leq i \leq n$, where

$$
\begin{aligned}
& \xi_{0, p}=\operatorname{span}\left\{e_{1}, \ldots, e_{n}, f_{1}, \ldots, f_{n}\right\} \\
& \xi_{1, p}=\operatorname{span}\left\{e_{1}, \ldots, e_{n}, g_{1}, \ldots, g_{n}\right\}
\end{aligned}
$$

are the canonical symplectic basis adapted to the orthogonal decompositions.

Step 3: Now we define $L_{p}$ for $p \in N_{\epsilon}(S)$. Given $p \in N_{\epsilon}(S) \backslash S$, pick a basis on $T_{p} \mathcal{H}=\operatorname{span}\left\{e_{1}, \ldots, e_{n-1}\right\}$, and let $e_{n}$ generate $T_{p} \mathcal{F} / T_{p} \mathcal{H} \simeq \mathbb{R}$. Let $\left(T_{p} \mathcal{F}\right)^{\perp_{0}}=\operatorname{span}\left\{f_{1}, \ldots, f_{n}\right\}$ be the canonical dual basis with respect to $d \alpha_{0}$, i.e., $d \alpha_{0}\left(e_{i}, f_{j}\right)=\delta_{i j}$ and all the other pairings vanish. Similarly let $\left(T_{p} \mathcal{F}\right)^{\perp_{1}}=\operatorname{span}\left\{g_{1}, \ldots, g_{n}\right\}$ with respect to $d \alpha_{1}$. Let $\chi(r):[0, \epsilon] \rightarrow$ $[0, \epsilon]$ be a smooth increasing function such that $\chi(r)=0$ for $r$ close to 0 and $\chi(r)=\epsilon$ for $r$ close to $\epsilon$. Denote $r_{p}$ the distance from $p$ to $S$. Finally we define $L_{p}\left(f_{i}\right)=g_{i}$ for $1 \leq i \leq n-1$, and

$$
L_{p}\left(\left(\epsilon-\chi\left(r_{p}\right)\right) X_{0}+\chi\left(r_{p}\right) f_{n}\right)=\left(\epsilon-\chi\left(r_{p}\right)\right) X_{1}+\chi\left(r_{p}\right) g_{n}
$$

for any $p \in N_{\epsilon}(S) \backslash S$. Our definition obviously extends to $S$ by asking $L_{p}\left(X_{0}\right)=X_{1}$ for $p \in S$.

One can check that the definition of $L_{p}$ is independent of the choice of various basis, well-defined on the overlaps, and depends smoothly on $p \in Y$. In particular we note that for small $\epsilon$, the vector $\left(\epsilon-\chi\left(r_{p}\right)\right) X_{0}+\chi\left(r_{p}\right) f_{n}$, together with $\left\{f_{1}, \ldots, f_{n-1}\right\}$, span $T_{p} M / T_{p} Y$. Similarly for $\left(\epsilon-\chi\left(r_{p}\right)\right) X_{1}+$ $\chi\left(r_{p}\right) g_{n}$.

Applying Theorem 3.3, we get a diffeomorphism $\psi: \mathcal{U}_{0} \rightarrow \mathcal{U}_{1}$ between neighborhoods of $Y$ which is fixed on $Y$, such that $\left.d \psi\right|_{Y}=L$. Abusing notations, we still use $\alpha_{1}$ to denote $\psi^{*}\left(\alpha_{1}\right)$. Observe that $\alpha_{0}=\alpha_{1}$ and $d \alpha_{0}=d \alpha_{1}$ on $Q$ by construction, but they do not necessarily agree on $N_{\epsilon}(S)$.

Claim 3.9. The form $\alpha_{t}=(1-t) \alpha_{0}+t \alpha_{1}$ is contact on $Y$, and therefore on a neighborhood of $Y$, for all $0 \leq t \leq 1$.

Proof of Claim 3.9. It is easy to see that $\alpha_{t}$ is contact along $S$ since $d \lambda_{0}=$ $d \lambda_{1}$ defines a positive area form in the normal plane to $S$. Therefore we only need to verify the contact condition on $N_{\epsilon}(S) \backslash S$. Let $\mathbf{n}$ be the unit normal vector field to $\mathcal{F}$ in $N_{\epsilon}(S) \backslash S$. To understand the pull-back contact 
structure $\alpha_{1}$ on $Y$, we need to solve for $L_{p}^{-1}\left(g_{n}\right)$ using (2.1.2). Observe that

$$
X_{0}=C_{1}\left(\mathbf{n}-\sum_{i=1}^{n}\left\langle\mathbf{n}, f_{i}\right\rangle f_{i}\right), \quad \text { and } X_{1}=C_{2}\left(\mathbf{n}-\sum_{i=1}^{n}\left\langle\mathbf{n}, g_{i}\right\rangle g_{i}\right),
$$

where $C_{1}=1 /\left\|\mathbf{n}-\sum_{i=1}^{n}\left\langle\mathbf{n}, f_{i}\right\rangle f_{i}\right\|, C_{2}=1 /\left\|\mathbf{n}-\sum_{i=1}^{n}\left\langle\mathbf{n}, g_{i}\right\rangle g_{i}\right\|$, and $\langle\cdot, \cdot\rangle$ denotes the inner product induced by $g$. Plug (3.2.4) into (3.2.3) and use the fact that $L_{p}(\mathbf{n})=\mathbf{n}$, we obtain for each $p \in N_{\epsilon}(S) \backslash S$

$$
B^{\prime} L_{p}^{-1}\left(g_{n}\right)=\sum_{i=1}^{n-1} A_{i}^{\prime} f_{i}+B^{\prime \prime} f_{n}+C^{\prime} \mathbf{n}
$$

where $A_{i}^{\prime}=\left(1-r_{p}\right) \sum_{i=1}^{n-1}\left\langle\mathbf{n}, C_{2} g_{i}-C_{1} f_{i}\right\rangle, B^{\prime}=C_{2}\left(\chi\left(r_{p}\right)-\left\langle\mathbf{n}, g_{n}\right\rangle\right), B^{\prime \prime}=$ $C_{1}\left(r_{p}-\left\langle\mathbf{n}, f_{n}\right\rangle\right)$, and $C^{\prime}=\left(C_{1}-C_{2}\right)\left(1-r_{p}\right)$. Now we choose small $\epsilon>0$ such that $\left\langle\mathbf{n}, f_{n}\right\rangle>2 \epsilon$ and $\left\langle\mathbf{n}, g_{n}\right\rangle>2 \epsilon$ for every $p \in N_{\epsilon}(S)$. This is possible because the two-form $d \lambda_{0}=d \lambda_{1}$ restricts to a positive area forms on the (co-oriented) normal plane to $S$.

By letting $A_{i}=A_{i}^{\prime} / B^{\prime}, B=B^{\prime \prime} / B^{\prime}$ and $C=C^{\prime} / B^{\prime}$, we get

$$
L_{p}^{-1}\left(g_{n}\right)=\sum_{i=1}^{n-1} A_{i} f_{i}+B f_{n}+C \mathbf{n}
$$

such that $B>0$. Therefore we have computed the symplectic basis

$$
\xi_{1}=\operatorname{span}\left\{e_{1}, \ldots, e_{n}, f_{1} \ldots, f_{n-1}, L_{p}^{-1}\left(g_{n}\right)\right\}
$$

with respect to the pull-back symplectic form $d \alpha_{1}$. Now a straightforward calculation using (3.2.5) verifies the assertion of Claim 3.9, and we leave the details to the interested reader.

Finally the standard Moser's technique, applied to the path $\alpha_{t}$, isotopes $\psi$ to a diffeomorphism $\phi: \mathcal{U}_{0} \rightarrow \mathcal{U}_{1}$ which satisfies all the desired properties.

Finally the conclusion of Theorem 1.4 is the combination of the conclusions of Lemma 3.1, Lemma 3.2, Lemma 3.6 and Lemma 3.8.

\section{References}

[1] C. Ehresmann, Les connexions infinitésimales dans un espace fibré différentiable. In: Séminaire Bourbaki, Vol. 1, Exp. No. 24, 153-168, Soc. Math. France, Paris (1995). 
[2] Y. Eliashberg, Classification of overtwisted contact structures on 3manifolds. Invent. Math., 98 (1989), no. 3, 623-637.

[3] Y. Eliashberg, Contact 3-manifolds twenty years since J. Martinet's work. Ann. Inst. Fourier (Grenoble), 42 (1992), no. 1-2, 165-192.

[4] J. B. Etnyre and D. M. Pancholi, On generalizing Lutz twists. J. Lond. Math. Soc. (2), 84 (2011), no. 3, 670-688.

[5] E. Giroux, Convexité en topologie de contact. Comment. Math. Helv., 66 (1991), no. 4, 637-677.

[6] Y. Huang, On Legendrian foliations in contact manifolds II: Deformation theory. Preprint.

[7] P. Massot, K. Niederkrüger and C. Wendl, Weak and strong fillability of higher dimensional contact manifolds. Invent. Math., 192 (2013), no. 2, 287-373.

[8] S. Morita, Geometry of characteristic classes. In: Translations of Mathematical Monographs, Vol. 199, American Mathematical Society, Providence, RI (2001), ISBN 0-8218-2139-3. Translated from the 1999 Japanese original, Iwanami Series in Modern Mathematics.

[9] K. Niederkrüger, The plastikstufe-a generalization of the overtwisted disk to higher dimensions. Algebr. Geom. Topol., 6 (2006) 2473-2508.

[10] K. Niederkrüger, On fillability of contact manifolds (2013). Habilitation.

[11] Y.-G. Oh and J.-S. Park, Deformations of coisotropic submanifolds and strong homotopy Lie algebroids. Invent. Math., 161 (2005), no. 2, 287360.

[12] F. Presas, A class of non-fillable contact structures. Geom. Topol., 11 (2007) 2203-2225.

[13] W. P. Thurston, Existence of codimension-one foliations. Ann. of Math. (2), 104 (1976), no. 2, 249-268.

MAX-PlanCK-INSTITUT FÜR MATHEMATIK

Vivatsgasse 7, Bonn 53111, Germany

E-mail address: yhuang@mpim-bonn.mpg.de

Received December 14, 2013 